\title{
Telegraphic processes with stochastic resetting
}

\author{
Jaume Masoliver* \\ Department of Condensed Matter Physics and Institute of Complex Systems (UBICS), University of Barcelona, Catalonia, Spain
}

(Received 4 September 2018; published 14 January 2019)

\begin{abstract}
We investigate the effects of resetting mechanisms on random processes that follow the telegrapher's equation instead of the usual diffusion equation. We thus study the consequences of a finite speed of signal propagation, the landmark of telegraphic processes. Likewise diffusion processes where signal propagation is instantaneous, we show that in telegraphic processes, where signal propagation is not instantaneous, random resettings also stabilize the random walk around the resetting position and optimize the mean first-arrival time. We also obtain the exact evolution equations for the probability density of the combined process and study the limiting cases.
\end{abstract}

DOI: 10.1103/PhysRevE.99.012121

\section{INTRODUCTION}

In recent years there has been some amount of work on the effects and applications of the combination of unbounded diffusion processes with a resetting mechanism which occasionally, at random instants of time, returns the process to a given fixed location. The random dynamics of the process and the resetting mechanism are taken to be independent of each other, while resettings to a given position occur at Poissonian times. Besides few antecedents in physics [1] and in the mathematics literature (see [2] for more information), the topic has been revived and further developed by the works of Evans, Majumdar, and collaborators [3-9] as well as an increasing number of different investigators of which we cite a very small sample $[2,10-15]$.

The interest in this kind of problem essentially resides on two rather remarkable facts. Firstly, the verification that resetting stabilizes the underlying process, in the sense that a nonstationary process becomes stationary when a resetting mechanism is implemented. Secondly, and surely of wider range, the fact that random resettings may significantly reduce the mean first-passage time which, in turn, optimizes any search process based on the combined process. The overall result is the universal character of resetting processes [13,14,16-18].

It has also been shown very recently that restart can optimize the probability of success in Bernouilli trials [14]. Due to the ubiquitous nature of Bernouilli trials in modeling countless phenomena, this optimization greatly enhances the relevance of stochastic resettings from theoretical as well as practical points of view. No wonder the problem has many applications in several branches of physical and social sciences and technology, with special emphasis on searching processes as, for instance, proteins in DNA [19-21], animal foraging $[22,23]$, or internet search algorithms which are instrumental in data mining [24-26], just to name a few.

The development of stochastic resettings has been mainly addressed when the primary process is a free diffusion

\footnotetext{
*jaume.masoliver@ub.edu
}

processes, that is to say, when the underlying process is described by the ordinary random walk for discrete time and space, or by Brownian motion for continuous time and space. To my knowledge there have been few exceptions to this line of research. These are (i) resettings within the continuoustime random-walk formalism [10], (ii) resettings when the underlying process is described by Lévy flights [12], and (iii) the effect of resettings on the stationary distribution of some potential fields of force [27].

In this paper we want to address the problem for another generalization in which the random walker has finite speed of propagation, so that the underlying process is driven by the telegrapher's equation instead of the ordinary diffusion equation. In this way we will analyze and quantify the effects of having a finite speed of propagation and also of ballistic fluxes when all of this is combined with a resetting mechanism.

The paper is organized as follows. In Sec. II we briefly explain the similarities and differences between diffusion and telegraphic processes. In Sec. III we describe the resetting mechanism and show that, under rather general circumstances, any nonstationary random process becomes stationary when resetting mechanisms are implemented. In Secs. IV and $\mathrm{V}$ we study the telegraphic process under resettings and obtain the forward equation (Sec. IV) and the backward equation (Sec. V) for the probability density function. In Sec. VI we address the first-passage problem obtaining the exact expressions for the survival probability and the mean first-arrival time and show the nonmonotonous character of the problem in terms of the resetting frequency. Concluding remarks are in Sec. VII and some technical details in the two appendices.

\section{DIFFUSION AND TELEGRAPHIC PROCESSES}

Suppose we have an unbounded diffusion process without any resetting mechanism. The probability density function (PDF), or propagator, $p\left(x, t \mid x_{0}\right)$, satisfies the diffusion equation

$$
\frac{\partial p}{\partial t}=D \frac{\partial^{2} p}{\partial x^{2}},
$$


where $D$ is the diffusion coefficient and we assume, for simplicity, one-dimensional processes, even though this can be easily extended to an arbitrary number of dimensions. Assuming that the process is initially at $x_{0}$ with certainty, that is, $p\left(x, 0 \mid x_{0}\right)=\delta\left(x-x_{0}\right)$, the solution to Eq. (1) is

$$
p\left(x, t \mid x_{0}\right)=\frac{1}{(4 \pi D t)^{1 / 2}} e^{-\left(x-x_{0}\right)^{2} / 4 D t} .
$$

Despite its universality, the diffusion equation has some shortcomings; one of them is the lack of a finite velocity of propagation. Indeed, looking at Eq. (2) we see that the propagator instantaneously spreads out from the initial density $\delta\left(x-x_{0}\right)$ to the Gaussian form given in (2). In consequence, there is a nonzero probability for the walker to be found outside the interval $\left|x-x_{0}\right|>c t$ where $c$ is the speed of light in the vacuum. In other words, there is a small albeit nonzero probability for a walker traveling faster than the speed of light. The diffusion equation is, therefore, not compatible with relativity $[28,29]$. Other shortcomings of the diffusion equation and, hence, of diffusion processes, may be summarized by its inability to account for ballistic motion which implies limitations in the description of early-time effects when the ballistic motion is not negligible, especially near interfaces. All of this is rather relevant in the transport of particles, especially when anisotropic scattering along the forward direction is taken into account [29-31].

The telegrapher's equation (TE) is one of the simplest relativistic generalizations of the diffusion equation still retaining diffusive properties but having a finite propagation speed as well as considering possible fluxes of ballistic motion. It is a hyperbolic type of partial differential equation in which the one-dimensional case reads

$$
\frac{\partial^{2} p}{\partial t^{2}}+2 \lambda \frac{\partial p}{\partial t}=v^{2} \frac{\partial^{2} p}{\partial x^{2}},
$$

where $\lambda^{-1}$ is a characteristic time and $v>0$ a characteristic speed. As $\lambda \rightarrow 0$ with $v$ fixed, TE becomes the wave equation,

$$
\frac{\partial^{2} p}{\partial t^{2}}=v^{2} \frac{\partial^{2} p}{\partial x^{2}}
$$

whereas as $\lambda \rightarrow \infty$ and $v \rightarrow \infty$ with $v^{2} /(2 \lambda) \rightarrow D$ finite it becomes the diffusion equation (1). Equation (3) thus possesses wave and diffusion properties and describes "diffusion with finite propagation speed" or "waves with damping" [29,32].

Different physical situations can result in telegraphic processes described by the TE. The equation first appeared in the 19th century with the works of Kelvin and Heaviside related to the analysis of transmission of electromagnetic waves in telegraph wires. In this context $\mathrm{TE}$ can be derived directly from Maxwell's equations [29,31]. It can also be phenomenologically derived from thermodynamics by a nonlocal generalization of Fick's law called Cattaneo's equation [33-35] $]^{1}$ as well as random-walk theory where the one-dimensional TE is

\footnotetext{
${ }^{1}$ There is, in addition, the interaction between thermodynamics and resetting processes, the so-called "stochastic thermodynamics of resettings," which has been recently addressed and discussed in Refs. [36] and [37].
}

the master equation of the persistent random walk [38,39] (see also [40] for a recent three-dimensional generalization and [41-43] for alternative derivations of hyperbolic equations).

From a mesoscopic point of view (somewhere in between the microscopic view of random-walk models and the macroscopic approach of thermodynamics) telegraphic processes are closely related to Brownian motion. As was studied some years ago in Refs. [44-46], the telegrapher's equation, like a diffusion equation, can also be derived from the ChapmanKolmogorov equation, the master equation for Markovian processes [47]. ${ }^{2}$

Contrary to a diffusion equation where only one initial condition on $p\left(x, t \mid x_{0}\right)$ is needed, the solution to TE (3) requires two initial conditions. These are [29]

$$
p\left(x, 0 \mid x_{0}\right)=\delta\left(x-x_{0}\right),\left.\quad \frac{\partial p}{\partial t}\right|_{t=0}=0 .
$$

For the sake of completeness we outline in Appendix A the derivation of the solution to TE (3) under initial conditions (5). Although this solution has been known since a very long time ago, its derivation remains quite obscure [29]. The exact solution reads

$$
\begin{aligned}
p\left(x, t \mid x_{0}\right)= & \frac{1}{2 v} e^{-\lambda t}\left\{\delta\left(t-\left|x-x_{0}\right| / v\right)\right. \\
& \left.+\lambda \Theta\left(t-\left|x-x_{0}\right| / v\right)\left[I_{0}(\lambda \tau)+(t / \tau) I_{1}(\lambda \tau)\right]\right\},
\end{aligned}
$$

where $\delta(\cdot)$ is Dirac's function, $\Theta(\cdot)$ the Heaviside's step function, $\tau=\tau(t, x)$ is the "proper time" defined by

$$
\tau=\sqrt{t^{2}-\left|x-x_{0}\right|^{2} / v^{2}}
$$

and $I_{0}(\cdot)$ and $I_{1}(\cdot)$ are modified Bessel functions.

The two major characteristics of the telegraphic processes mentioned above are apparent from Eq. (6). First, there is a delta function term decaying exponentially with time. In transport theory this term corresponds to particles that do not change their motion and follow ballistic motion. Because of the decaying exponential the effect of the delta pulse fades away as time increases. Let us incidentally note that in the wave limit the PDF given in Eq. (6) describes deterministic motion. Indeed, setting $\lambda=0$ in Eq. (6) we get

$$
p\left(x, t \mid x_{0}\right)=\frac{1}{2 v} \delta\left(t-\left|x-x_{0}\right| / v\right),
$$

$(\lambda \rightarrow 0)$ which corresponds to the deterministic motion of a delta pulse from its initial location $x_{0}$ to a final location $x$ at time $t=\left|x-x_{0}\right| / v$.

The second feature in the solution given by Eq. (6) is the presence of the step function $\Theta\left(t-\left|x-x_{0}\right| / v\right)$ which excludes diffusion outside the interval $\left|x-x_{0}\right|>v t$. This

\footnotetext{
${ }^{2}$ It is worth noticing that such a derivation is obtained by retaining quadratic terms in the time expansion of the Chapman-Kolmogorov equation which sets a characteristic timescale and a characteristic velocity. The Markovian character of the process is assured for times greater than the characteristic time while, to my knowledge, a possible non-Markovian character for smaller times is still an unsettled question [46].
} 
is a manifestation of the property of finite speed of signal propagation and makes TE a relativistic equation as opposed to the diffusion equation which is not.

\section{THE RESETTING MECHANISM}

Let $X(t)$ be a random process on the line which, starting initially at $x_{0}$, suffers resettings to some fixed position $x_{r}$. Resettings occur at random times and we denote by $\phi(\tau)$ the PDF of the time interval between two consecutive resettings. ${ }^{3}$ For the rest of this work we will assume that resetting times are Poissonian, so that

$$
\phi(\tau)=r e^{-r \tau}
$$

here $r>0$ is the rate of resetting, so that $r^{-1}$ is the mean time between two consecutive resetting events. Before proceeding further let us note that in this Poissonian case the probability that no resettings occur for time intervals greater than $\tau$ is

$$
\int_{\tau}^{\infty} \phi\left(\tau^{\prime}\right) d \tau^{\prime}=e^{-r \tau}
$$

Let us denote by $p\left(x, t \mid x_{0}, t_{0}\right)$ the propagator of $X(t)$ :

$p\left(x, t \mid x_{0}, t_{0}\right) d x=\operatorname{Prob}\left\{x<X(t) \leqslant x+d x \mid X\left(t_{0}\right)=x_{0}\right\}$.

This propagator for the entire process (i.e., including resettings) can be written in terms of the propagator with no resetting events which we denote by $p_{0}\left(x, t \mid x_{0}, t_{0}\right)$. Indeed, observe that the probability for a random walker to travel from $x_{0}$ to $x$ is the sum of the probability of traveling when no reset has occurred plus the probability of traveling from the resetting position $x_{r}$ to $x$ after the last reset event. These considerations lead to the following renewal equation $[8,10,12]$ :

$$
\begin{aligned}
p\left(x, t \mid x_{0}, t_{0}\right)= & e^{-r\left(t-t_{0}\right)} p_{0}\left(x, t \mid x_{0}, t_{0}\right) \\
& +r \int_{t_{0}}^{t} e^{-r\left(t-t^{\prime}\right)} p_{0}\left(x, t \mid x_{r}, t^{\prime}\right) d t^{\prime},
\end{aligned}
$$

where the first term on the right-hand side accounts for the probability density when no reset event has occurred up to time $t$. The second term represents the probability density that the last resetting occurred at any intermediate time $t^{\prime}$.

In what follows we will assume that the underlying (i.e., reset-free) process is time homogeneous implying that its propagator only depends on time differences, $p_{0}\left(x, t \mid x_{0}, t_{0}\right)=$ $p_{0}\left(x, t-t_{0} \mid x_{0}\right)$. In the present case $p_{0}$ obeys TE (3) which is indeed invariant under time translations. Using this property we easily see from Eq. (9) that the complete process including resettings is also time homogeneous, that is,

$$
p\left(x, t \mid x_{0}, t_{0}\right)=p\left(x, t-t_{0} \mid x_{0}\right)
$$

\footnotetext{
${ }^{3}$ It is implicit in this formulation that resettings occur instantaneously, that is to say, at any random instant of time $t_{i}$ the transition $X\left(t_{i}\right) \rightarrow x_{r}$ is instantaneous. Obviously this is unphysical since it implies an infinite speed for such a transition. In dealing with telegraphic processes we will assume in practice that the transition velocity is much greater than the signal speed of the telegraphic process.
}

which allows us to take $t_{0}=0$ without loss of generality and write Eq. (9) in simpler form:

$$
p\left(x, t \mid x_{0}\right)=e^{-r t} p_{0}\left(x, t \mid x_{0}\right)+r \int_{0}^{t} e^{-r t^{\prime}} p_{0}\left(x, t^{\prime} \mid x_{r}\right) d t^{\prime} .
$$

Let us now address the important question of the stationarity of the complete process. To this end we define the stationary distribution as the long-time limit of the (timehomogeneous) propagator [47]:

$$
p_{\mathrm{st}}(x)=\lim _{t \rightarrow \infty} p\left(x, t \mid x_{0}\right),
$$

provided that the limit is finite and different from zero. Taking this limit in Eq. (10) we get

$$
p_{\text {st }}(x)=r \int_{0}^{\infty} e^{-r t} p_{0}\left(x, t \mid x_{r}\right) d t,
$$

the expression previously obtained in [7] and [12] which shows an important property of the resetting mechanism, that is, the possible existence of a stationary state even if in the absence of resettings the underlying process is not stationary [in other words, when the limit (11) on $p_{0}$ does not exist or it is zero]. Note that the only condition for this to happen is the finiteness of the integral in Eq. (12). This fact is even more clearly seen in terms of the Laplace transform of the free propagator,

$$
\hat{p}_{0}\left(x, s \mid x_{0}\right)=\int_{0}^{\infty} e^{-s t} p_{0}\left(x, t \mid x_{0}\right) d t,
$$

which proves that the stationary distribution is simply given by

$$
p_{\text {st }}(x)=r \hat{p}_{0}\left(x, r \mid x_{r}\right),
$$

showing the explicit dependence of the stationary state on the resetting location $x_{r}$ and the resetting rate $r$. In consequence the existence of a stationary density for the combined process depends on the existence of the Laplace transform of the resetfree process.

We thus see that resettings can turn a nonstationary process into a stationary one. This is, for instance, the case of Brownian motion which is not stationary but that Poisson resetting events turn it into a stationary process. In effect, in this case the underlying propagator $p_{0}\left(x, t \mid x_{0}\right)$ is given by Eq. (2) which taking into account the Laplace transform [48]

$$
\mathcal{L}\left\{\frac{e^{-a^{2} / 4 t}}{\sqrt{\pi t}}\right\}=\frac{e^{-|a| \sqrt{s}}}{\sqrt{s}}
$$

leads, by way of Eq. (13), to a tent-shape distribution (i.e., the Laplace density) around the resetting location $x_{r}$ :

$$
p_{\mathrm{st}}(x)=\frac{1}{2} \sqrt{\frac{r}{D}} e^{-\left|x-x_{r}\right| \sqrt{r / D}} .
$$

Note that $p_{\mathrm{st}}(x) \rightarrow 0$ as $r \rightarrow 0$ (lack of stationarity of the Brownian motion without resettings). Also $p_{\mathrm{st}}(x) \rightarrow \delta(x-$ $\left.x_{r}\right)$ as $r \rightarrow \infty$ and the stationary density becomes more and more peaked around $x_{r}$ with increasing reset rates.

We, therefore, see the otherwise rather intuitive fact that the resetting mechanism stabilizes the Brownian motion around 
the stationary mean value given by $x_{r}$ with stationary variance $\sigma_{\mathrm{st}}^{2}=D / r$.

\section{TELEGRAPHIC PROCESSES WITH RESETTINGS}

We now address the main objective of this paper and suppose that the underlying process with no resetting events is a telegraphic process. In such case the PDF $p_{0}\left(x, t \mid x_{0}\right)$ satisfies TE (3) with initial conditions (5):

$$
\begin{gathered}
\frac{\partial^{2} p_{0}}{\partial t^{2}}+2 \lambda \frac{\partial p_{0}}{\partial t}=v^{2} \frac{\partial^{2} p_{0}}{\partial x^{2}} \\
p_{0}\left(x, 0 \mid x_{0}\right)=\delta\left(x-x_{0}\right),\left.\quad \frac{\partial p_{0}}{\partial t}\right|_{t=0}=0 .
\end{gathered}
$$

Our first goal will be obtaining the evolution equation for the complete propagator $p\left(x, t \mid x_{0}\right)$ when Poissonian resetting events are superposed to the telegraphic process. ${ }^{4}$ The starting point is the renewal equation [cf. Eq. (10)]

$$
p\left(x, t \mid x_{0}\right)=e^{-r t} p_{0}\left(x, t \mid x_{0}\right)+r \int_{0}^{t} e^{-r t^{\prime}} p_{0}\left(x, t^{\prime} \mid x_{r}\right) d t^{\prime} .
$$

The time derivative of this equation yields

$$
\frac{\partial p}{\partial t}=e^{-r t} \frac{\partial p_{0}}{\partial t}+r e^{-r t}\left[p_{0}^{(r)}-p_{0}\right] .
$$

Here and in the rest of the paper we use the shorthand notation

$$
p_{0}=p_{0}\left(x, t \mid x_{0}\right) \quad \text { and } \quad p_{0}^{(r)}=p_{0}\left(x, t \mid x_{r}\right)
$$

and similarly for $p$ and $p^{(r)}$. Setting $x_{0}=x_{r}$ in Eq. (17) we see that

$$
p^{(r)}=e^{-r t} p_{0}^{(r)}+r \int_{0}^{t} e^{-r t^{\prime}} p_{0}\left(x, t^{\prime} \mid x_{r}\right)
$$

Subtracting Eqs. (19) and (17) the integrals cancel and we get

$$
e^{-r t}\left[p_{0}^{(r)}-p_{0}\right]=p^{(r)}-p,
$$

which substituting back into Eq. (18) yields

$$
\frac{\partial p}{\partial t}=e^{-r t} \frac{\partial p_{0}}{\partial t}+r\left[p^{(r)}-p\right]
$$

Taking the second time derivative we have

$$
\frac{\partial^{2} p}{\partial t^{2}}=-r e^{-r t} \frac{\partial p_{0}}{\partial t}+e^{-r t} \frac{\partial^{2} p_{0}}{\partial t^{2}}+r\left[\frac{\partial p^{(r)}}{\partial t}-\frac{\partial p}{\partial t}\right],
$$

and using Eq. (21) we obtain

$$
\frac{\partial^{2} p}{\partial t^{2}}=e^{-r t} \frac{\partial^{2} p_{0}}{\partial t^{2}}-2 r \frac{\partial p}{\partial t}+r \frac{\partial p^{(r)}}{\partial t}+r^{2}\left[p^{(r)}-p\right] .
$$

\footnotetext{
${ }^{4}$ Obtaining the evolution equation of the complete propagator is not really a necessary step in order to know the solution. Indeed, the expression for $p\left(x, t \mid x_{0}\right)$ can be readily obtained after substituting into the renewal equation (10) the exact expression of the resetfree propagator $p_{0}\left(x, t \mid x_{0}\right)$ given in Eq. (6). However, knowing the equation for $p$ is worthy by itself but also because it provides a guide for obtaining the backward equation which is instrumental in our approach to the first-passage problem, as we will see in the forthcoming sections.
}

On the other hand, the second derivative of Eq. (17) with respect to $x$ yields

$$
\frac{\partial^{2} p}{\partial x^{2}}=e^{-r t} \frac{\partial^{2} p_{0}}{\partial x^{2}}+r \int_{0}^{t} e^{-t^{\prime}} \frac{\partial^{2} p_{0}^{(r)}}{\partial x^{2}} d t^{\prime} .
$$

By combining these derivatives [i.e., Eqs. (21), (22), and (23)] we show in Appendix B that the complete propagator satisfies the following inhomogeneous TE with a source term at the resetting position:

$$
\frac{\partial^{2} p}{\partial t^{2}}+2(\lambda+r) \frac{\partial p}{\partial t}=v^{2} \frac{\partial^{2} p}{\partial x^{2}}+r(r+2 \lambda)\left[\delta\left(x-x_{r}\right)-p\right]
$$

which constitutes one of the main results of this section.

The initial conditions that accompany Eq. (24) can be obtained from the initial conditions of the free propagator $p_{0}$. Indeed setting $t=0$ in the renewal equation (10) we see that $p\left(x, 0 \mid x_{0}\right)=p_{0}\left(x, 0 \mid x_{0}\right)$ and from Eq. (16) we get

$$
p\left(x, 0 \mid x_{0}\right)=\delta\left(x-x_{0}\right) .
$$

The second initial condition is obtained from Eq. (21) which, after setting $t=0$, reads

$$
\left.\frac{\partial p}{\partial t}\right|_{t=0}=\left.\frac{\partial p_{0}}{\partial t}\right|_{t=0}+r\left[p\left(x, 0 \mid x_{r}\right)-p\left(x, 0 \mid x_{0}\right)\right],
$$

but from Eq. (25) we see that $p\left(x, 0 \mid x_{r}\right)=\delta\left(x-x_{r}\right)$ and $p\left(x, 0 \mid x_{0}\right)=\delta\left(x-x_{0}\right)$, hence [see also Eq. (16)]

$$
\left.\frac{\partial p}{\partial t}\right|_{t=0}=r\left[\delta\left(x-x_{r}\right)-\delta\left(x-x_{0}\right)\right] .
$$

We have shown in Sec. III that in general resettings stabilize any nonstationary random process. This is obviously the case of telegraphic processes. Indeed, the reset-free process is not stationary as can be seen directly from Eq. (11) after using a well-known property of the Laplace transform [48] and Eq. (A2):

$$
p_{0}^{(\mathrm{st})}(x)=\lim _{t \rightarrow \infty} p_{0}\left(x, t \mid x_{0}\right)=\lim _{s \rightarrow 0}\left[s \hat{p}_{0}\left(x, s \mid x_{0}\right)\right]=0 .
$$

On the other hand, the combined process with Poissonian resettings is stationary. In effect, we know that the stationary density of the complete process is related to the Laplace transform of the reset-free density by Eq. (13), $p_{\mathrm{st}}(x)=$ $r \hat{p}_{0}\left(x, r \mid x_{r}\right)$. For telegraphic processes $\hat{p}_{0}\left(x, s \mid x_{0}\right)$ is given in Eq. (A2) and we obtain the following Laplace density as the stationary PDF: 5

$$
p_{\mathrm{st}}(x)=\frac{1}{2 v} \sqrt{r^{2}+2 \lambda r} \exp \left\{-\left|x-x_{r}\right| \sqrt{r^{2}+2 \lambda r} / v\right\} .
$$

Therefore, as in Brownian motion, resettings stabilize the telegraphic process around the average value $x_{r}$ with the stationary variance $\sigma_{\text {stat }}^{2}=v^{2} /\left(r^{2}+2 \lambda r\right)$.

${ }^{5}$ Note that the stationary density (27) can also be obtained by solving Eq. (24) with initial conditions (25) and (26) and then taking the limit $t \rightarrow \infty$. Obviously the result of both procedures is the same. 
Let us finish this section showing the diffusion limit and the wave limit of TE (24).

(i) In order to obtain the diffusive limit we rewrite Eq. (24) as

$$
\begin{aligned}
\frac{1}{2 \lambda} \frac{\partial^{2} p}{\partial t^{2}}+\left(1+\frac{r}{\lambda}\right) \frac{\partial p}{\partial t}= & \frac{v^{2}}{2 \lambda} \frac{\partial^{2} p}{\partial x^{2}}+r\left(1+\frac{r}{2 \lambda}\right) \\
& \times\left[\delta\left(x-x_{r}\right)-p\right] .
\end{aligned}
$$

In the diffusive limit, $\lambda \rightarrow \infty, v \rightarrow \infty$ and $v^{2} /(2 \lambda) \rightarrow D$, we get

$$
\frac{\partial p}{\partial t}=D \frac{\partial^{2} p}{\partial x^{2}}+r\left[\delta\left(x-x_{r}\right)-p\right],
$$

which is the resetting diffusion equation previously obtained in the literature [3]. In an analogous way, it is an easy check for the reader to obtain from Eq. (27) the stationary density in the diffusive limit [cf. Eq. (14)]

$$
p_{\text {st }}^{(\text {dif })}(x)=\frac{1}{2} \sqrt{r / D} e^{-\left|x-x_{r}\right| / \sqrt{r / D}} .
$$

(ii) In the wave limit ( $\lambda \rightarrow 0$ and $v$ finite) the inhomogeneous TE (24) reduces to a slightly simpler equation of the same kind

$$
\frac{\partial^{2} p}{\partial t^{2}}+2 r \frac{\partial p}{\partial t}=v^{2} \frac{\partial^{2} p}{\partial x^{2}}+r^{2}\left[\delta\left(x-x_{r}\right)-p\right]
$$

with the same characteristic velocity $v$ but with a time parameter $r^{-1}$ which coincides with the average time between consecutive resettings.

Let us incidentally note that in the presence of resettings the process in the wave limit is no longer deterministic because of the stochastic resetting events. In this case the stationary distribution is given by Eq. (27) with $\lambda=0$ :

$$
p_{\text {st }}^{(\mathrm{w})}(x)=(r / 2 v) e^{-r\left|x-x_{r}\right| / v} .
$$

If we compare this with the stationary distribution in the diffusion limit, Eq. (29), we see that both distributions are tent-shape with mean given by $x_{r}$ and variance (indicating the spread of distances from $x_{r}$ ) given by

$$
\sigma_{\mathrm{st}}^{2}=\frac{D}{r} \text { (diffusion), } \sigma_{\mathrm{st}}^{2}=\frac{v^{2}}{r^{2}} \text { (wave). }
$$

The stationary variance decreases faster with $r$ in the wave limit than in the diffusive limit. This different behavior can be easily interpreted on physical grounds. For in the wave limit randomness comes only from stochastic resettings while in the diffusive limit there are two sources of randomness, one coming from resettings but an additional one from the random evolution between consecutive resettings given by Brownian motion.

\section{THE BACKWARD EQUATION}

Our second major objective is to study the effects of resettings on the first-passage problem. We will approach this problem through the backward TE in the presence of Poissonian resettings. Before proceeding further let us observe that obtaining the the backward equation for the propagator is an interesting illustration on its own because the backward formalism for the telegrapher's equation is rather unknown. However, the backward equation for the propagator it is not strictly necessary for solving the first-passage problem since as proved in Ref. [9] one can obtain a renewal equation for the survival probability which allows one to get the mean first-passage time.

To deal with the backward problem we start from the renewal equation in the form given by Eq. (9):

$$
\begin{aligned}
p\left(x, t \mid x_{0}, t_{0}\right)= & e^{-r\left(t-t_{0}\right)} p_{0}\left(x, t \mid x_{0}, t_{0}\right) \\
& +r \int_{t_{0}}^{t} e^{-r\left(t-t^{\prime}\right)} p_{0}\left(x, t \mid x_{r}, t^{\prime}\right) d t^{\prime},
\end{aligned}
$$

where $p_{0}\left(x, t \mid x_{0}, t_{0}\right)$ satisfies the backward (also called adjoint) TE [49]

$$
\frac{\partial^{2} p_{0}}{\partial t_{0}^{2}}-2 \lambda \frac{\partial p_{0}}{\partial t_{0}}=v^{2} \frac{\partial^{2} p_{0}}{\partial x_{0}^{2}}
$$

with "final conditions"

$$
p_{0}\left(x, t \mid x_{0}, t\right)=\delta\left(x-x_{0}\right),\left.\quad \frac{\partial p_{0}}{\partial t_{0}}\right|_{t_{0}=t}=0 .
$$

In order to obtain the backward equation for the complete propagator $p\left(x, t \mid x_{0}, t_{0}\right)$, we take the derivative of Eq. (32) with respect to $t_{0}$ and proceed similarly as in the forward problem of the previous section. We get

$$
\frac{\partial p}{\partial t_{0}}=e^{-r\left(t-t_{0}\right)} \frac{\partial p_{0}}{\partial t_{0}}+r\left[p-p^{(r)}\right]
$$

The second derivative and the use of (35) yields

$$
\frac{\partial^{2} p}{\partial t_{0}^{2}}=e^{-r\left(t-t_{0}\right)} \frac{\partial^{2} p_{0}}{\partial t_{0}^{2}}+2 r \frac{\partial p}{\partial t_{0}}-r \frac{\partial p^{(r)}}{\partial t_{0}}-r^{2}\left[p-p^{(r)}\right] .
$$

On the other hand, from Eq. (32) we see that the second derivative of $p$ with respect to $x_{0}$ reads

$$
\frac{\partial^{2} p}{\partial x_{0}^{2}}=e^{-r\left(t-t_{0}\right)} \frac{\partial^{2} p_{0}}{\partial x_{0}^{2}} .
$$

By combining these derivatives as we have done in the previous section to obtain the forward equation (24) and taking also into account that the reset-free propagator $p_{0}$ satisfies the backward TE (33) we can readily obtain the following backward equation for the propagator $p$ of the whole process:

$$
\begin{aligned}
& \frac{\partial^{2} p}{\partial t_{0}^{2}}-2(\lambda+r) \frac{\partial p}{\partial t_{0}} \\
& =v^{2} \frac{\partial^{2} p}{\partial x_{0}^{2}}-r \frac{\partial p^{(r)}}{\partial t_{0}}+r(r+2 \lambda)\left[p^{(r)}-p\right] .
\end{aligned}
$$

The final conditions that accompany this equation are obtained from the final conditions on $p_{0}\left(x, t \mid x_{0}\right)$, Eq. (34), and from Eqs. (32) and (35). They are

$$
\begin{gathered}
p\left(x, t \mid x_{0}, t\right)=\delta\left(x-x_{0}\right), \\
\left.\frac{\partial p}{\partial t_{0}}\right|_{t_{0}=t}=r\left[\delta\left(x-x_{0}\right)-\delta\left(x-x_{r}\right)\right] .
\end{gathered}
$$

The backward equation (38) can be written in an alternative and somewhat simpler form which turns out to be 
very convenient for the study of first-passage problems. Indeed, taking into account time homogeneity, $p\left(x, t \mid x_{0}, t_{0}\right)=p\left(x, t-t_{0} \mid x_{0}\right)$, the change of timescale $t-t_{0} \rightarrow t$ (meaning that $t_{0}=0$ and $\partial / \partial t_{0} \rightarrow-\partial / \partial t$ and $\partial^{2} / \partial t_{0}^{2} \rightarrow \partial^{2} / \partial t^{2}$ ) turns Eq. (38) into the alternative form

$$
\begin{aligned}
& \frac{\partial^{2} p}{\partial t^{2}}+2(\lambda+r) \frac{\partial p}{\partial t} \\
& =v^{2} \frac{\partial^{2} p}{\partial x_{0}^{2}}+r \frac{\partial p^{(r)}}{\partial t}+r(r+2 \lambda)\left[p^{(r)}-p\right]
\end{aligned}
$$

with initial conditions

$$
\begin{gathered}
p\left(x, 0 \mid x_{0}\right)=\delta\left(x-x_{0}\right), \\
\left.\frac{\partial p}{\partial t}\right|_{t=0}=-r\left[\delta\left(x-x_{0}\right)-\delta\left(x-x_{r}\right)\right] .
\end{gathered}
$$

Let us note that in the diffusive limit $(\lambda \rightarrow \infty, v \rightarrow$ $\infty, v^{2} / 2 \lambda \rightarrow D$ ) Eq. (39) reduces to the inhomogeneous backward diffusion equation previously obtained by Evans and Majumdar [3]:

$$
\frac{\partial p}{\partial t}=D \frac{\partial^{2} p}{\partial x_{0}^{2}}-r\left[p-p^{(r)}\right] .
$$

In the wave limit $(\lambda \rightarrow 0)$ Eq. (39) reduces to the following backward and inhomogeneous TE:

$$
\frac{\partial^{2} p}{\partial t^{2}}+2 r \frac{\partial p}{\partial t}=v^{2} \frac{\partial^{2} p}{\partial x_{0}^{2}}+r \frac{\partial p^{(r)}}{\partial t}-r^{2}\left[p-p^{(r)}\right] .
$$

\section{THE FIRST-PASSAGE PROBLEM}

We next address the problem of characterizing when the telegraphic process with a reset mechanism first reaches a given value $x_{c}$, usually called critical value or threshold. This is called the first-passage problem and one of its main magnitudes is the first-passage time, defined to be the minimum time when the process first reaches $x_{c}$. This time is obviously a random variable depending on the given realization of the process. We will show that for the reset-free process the mean first-passage time is infinite while in the presence of Poissonian resettings this average time is finite and presents a minimum value as a function of the resetting frequency $r$.

The more direct way of solving the first-passage problem is obtaining the so-called survival probability which needs the specification of boundary conditions $[47,50]$. Let us first briefly discuss this delicate issue.

In the transport of particles, such as it appears, for instance, in chemical physics, the problem of survival is closely related to the question of when the particle is absorbed (and, hence, disappears) if it reaches a certain critical value $x_{c}$. For diffusion processes on the line, absorption at $x_{c}$ corresponds to $p\left(x_{c}, t \mid x_{0}\right)=0$ or $p\left(x, t \mid x_{c}\right)=0$. That is, if the particle either reaches $x_{c}$ or starts at $x_{c}$ it disappears. For telegraphic processes (and in the context of particle transport, at least for one-dimensional processes) the situation is more complex because of the property of persistence inherent in the telegrapher's equation [38]. In this context persistence, which is analogous to the physical property of momentum, makes it necessary, in deriving boundary conditions for absorption, to take into account the direction in which the particle is traveling. For if the particle starts at $x_{c}$ (or at time $t$ reaches $x_{c}$ ) it will disappear (i.e., it will be absorbed) only if the direction of the velocity is the appropriate one, otherwise the particle will escape. We studied this situation some years ago [49,51] and refer the reader to these works for more information.

However, within the scope of this paper, we are not primarily interested in the escape out of some interval (which implies absorption at both ends of the interval) but only on the first arrival time (FAT) to some particular value $x_{c}$. Let us note that if one starts at $x_{c}$, the FAT is zero, which implies that in dealing with this problem we should take the usual boundary condition $p\left(x, t \mid x_{c}\right)=0$, regardless of the direction of the velocity at this particular initial location. ${ }^{6}$

We define the survival probability $S\left(t \mid x_{0}\right)$ inside some interval $(a, b)$ as the probability that at time $t$ the process starting initially in the interval has not reached the boundary points $a$ and $b$ at time $t$ or before. This probability can be written as

$$
S\left(t \mid x_{0}\right)=\int_{a}^{b} p\left(x, t \mid x_{0}\right) d x
$$

$\left(a \leqslant x_{0} \leqslant b\right)$ where $p\left(x, t \mid x_{0}\right)$ is the propagator with appropriate boundary conditions at both ends of the interval [47]. For our problem (i.e., obtaining the first arrival time to either $a$ or $b)$ these boundary conditions are $p(x, t \mid a)=p(x, t \mid b)=$ 0 which, in turn, imply the boundary conditions $S(t \mid a)=$ $S(t \mid b)=0$.

In what follows we will restrict ourselves to only one threshold $x_{c}$. In such case we have the probabilities

$$
S\left(t \mid x_{0}\right)=\int_{-\infty}^{x_{c}} p\left(x, t \mid x_{0}\right) d x \text { if } x_{0}<x_{c},
$$

or

$$
S\left(t \mid x_{0}\right)=\int_{x_{c}}^{\infty} p\left(x, t \mid x_{0}\right) d x \text { if } x_{0}>x_{c} .
$$

As is well known [47], in terms of $S\left(t \mid x_{0}\right)$ the mean firstarrival time (MFAT) to some critical value $x_{c}$ is given by

$$
T\left(x_{0}\right)=\int_{0}^{\infty} S\left(t \mid x_{0}\right) d t .
$$

If we denote by $\hat{S}\left(s \mid x_{0}\right)$ the Laplace transform

$$
\hat{S}\left(s \mid x_{0}\right)=\int_{0}^{\infty} e^{-s t} S\left(t \mid x_{0}\right) d t
$$

then the MFAT is simply given by

$$
T\left(x_{0}\right)=\hat{S}\left(0 \mid x_{0}\right) .
$$

\footnotetext{
${ }^{6}$ Thus, for example, within the persistent random-walk approach if a particle moving to the right reaches some threshold $x_{c}$ the particle will not get trapped and disappear if at that instant of time the velocity of the particle changes direction; when this is the case the particle will go to the left and escape. If, however, we are only interested in whether the particle gets the mark $x_{c}$ regardless of any trapping, then any switch in velocity is irrelevant. If we define survival as not having touched $x_{c}$, then the survival probability at $x_{c}$ is zero, which is the boundary condition used in this paper.
} 


\section{A. Survival probability}

Let us return to the telegraphic process with resettings and integrate the backward equation (39) for the propagator with respect to the final position [cf. Eqs. (45)-(46)]. Commuting derivatives and integrals we readily see that the survival probability satisfies the following inhomogeneous telegrapher's equation:

$$
\begin{aligned}
& \frac{\partial^{2} S}{\partial t^{2}}+2(\lambda+r) \frac{\partial S}{\partial t} \\
& \quad=v^{2} \frac{\partial^{2} S}{\partial x_{0}^{2}}+r \frac{\partial S^{(r)}}{\partial t}-r(2 \lambda+r)\left[S-S^{(r)}\right],
\end{aligned}
$$

where $S^{(r)}=S\left(t \mid x_{r}\right)$. From Eqs. (40) and (41) and Eqs. (45) and (46) we see that the initial conditions are

$$
S\left(0 \mid x_{0}\right)=1,\left.\quad \frac{\partial S}{\partial t}\right|_{t=0}=0,
$$

while as a boundary condition we have

$$
S\left(t \mid x_{c}\right)=0 .
$$

The problem posed by Eqs. (49)-(51) becomes much simpler if we take the Laplace transform. Thus, bearing in mind the standard rules [cf. Eq. (50)]

$$
\mathcal{L}\left\{\frac{\partial S}{\partial t}\right\}=s \hat{S}\left(s \mid x_{0}\right)-1, \quad \mathcal{L}\left\{\frac{\partial^{2} S}{\partial t^{2}}\right\}=s^{2} \hat{S}\left(s \mid x_{0}\right)-s,
$$

we see after some elementary manipulations that $\hat{S}\left(s \mid x_{0}\right)$ obeys the following ordinary differential equation

$$
\frac{d^{2} \hat{S}}{d x_{0}^{2}}-\rho^{2}(s) \hat{S}=-\frac{\rho^{2}(s)}{r+s}\left[1+r \hat{S}^{(r)}\right]
$$

with boundary condition

$$
\hat{S}\left(s \mid x_{c}\right)=0 .
$$

Function $\rho(s)$ in Eq. (52) is defined by

$$
\rho(s)=\frac{1}{v} \sqrt{(r+s)(r+s+2 \lambda)},
$$

and $\hat{S}^{(r)}=\hat{S}\left(s \mid x_{r}\right)$ refers to the survival probability starting at the resetting point.

As can be easily seen by direct substitution, the solution to Eq. (52) that is finite for all possible values of $x_{0}$ (that is, even if $x_{0} \rightarrow \pm \infty$ ) and satisfying the boundary condition (53) is

$$
\hat{S}\left(s \mid x_{0}\right)=\frac{1}{r+s}\left[1+r S^{(r)}\right]\left[1-e^{-\rho(s)\left|x_{0}-x_{c}\right|}\right] .
$$

Setting $x_{0}=x_{r}$ we have

$$
\hat{S}^{(r)}=\frac{1}{r+s}\left[1+r S^{(r)}\right]\left[1-e^{-\rho(s)\left|x_{r}-x_{c}\right|}\right] .
$$

Solving for $S^{(r)}$ we have

$$
\hat{S}^{(r)}=\frac{1-e^{-\rho(s)\left|x_{r}-x_{c}\right|}}{s+r e^{-\rho(s)\left|x_{r}-x_{c}\right|}}
$$

and

$$
\frac{1}{r+s}\left[1+r S^{(r)}\right]=\frac{1}{s+r e^{-\rho(s)\left|x_{r}-x_{c}\right|}}
$$

Substituting this expression into Eq. (55) we finally get

$$
\hat{S}\left(s \mid x_{0}\right)=\frac{1-e^{-\rho(s)\left|x_{0}-x_{c}\right|}}{s+r e^{-\rho(s)\left|x_{r}-x_{c}\right|}} .
$$

\section{B. Mean first-arrival time}

Setting $s=0$ we obtain the MFAT [cf. Eq. (48)] ${ }^{7}$

$$
T\left(x_{0}\right)=\frac{1}{r} e^{\rho_{0}\left|x_{r}-x_{c}\right|}\left[1-e^{-\rho_{0}\left|x_{0}-x_{c}\right|}\right],
$$

where $\rho_{0}=\rho(0)$, that is [cf. Eq. (54)],

$$
\rho_{0}=\frac{1}{v} \sqrt{r(2 \lambda+r)} .
$$

We next analyze one of the most essential features of resettings, that is, the fact that the MFAT can be modulated by the resetting mechanism. In other words, $T\left(x_{0}\right)$ is a nonmonotonous function of the resetting rate $r$, decreasing for increasing values of $r$ toward a minimum value at a critical rate, $r_{\min }$, by then increasing for greater values of $r$.

Let us first note from Eq. (57) that for the telegraphic process with no resettings (i.e., $r=0$ ) the MFAT is infinite. We can be more precise by expanding Eqs. (57) and (58) in powers of the resetting rate $r$. We have

$$
T\left(x_{0}\right)=\frac{\sqrt{2 \lambda} / v}{\sqrt{r}}\left|x_{0}-x_{c}\right|+O(1),
$$

and the MFAT diverges as $r^{-1 / 2}$ when $r \rightarrow 0$ (we will see below that the limits $r \rightarrow 0$ and $\lambda \rightarrow 0$ do not commute).

On the other hand, when $r \rightarrow \infty$ we see from Eq. (58) that

$$
\rho_{0}=(r / v)[1+O(1 / r)] \text {. }
$$

Hence $e^{-\rho_{0}\left|x_{r}-x_{0}\right|}$ is exponentially small as $r \rightarrow \infty$ and the MFAT diverges exponentially,

$$
T\left(x_{0}\right) \simeq \frac{1}{r} e^{r\left|x_{r}-x_{c}\right| / v} \longrightarrow \infty \quad(r \rightarrow \infty) .
$$

We thus see that the MFAT to an arbitrary threshold $x_{c}$ diverges for both small and large values of the resetting frequency. Since, as we see from Eq. (57), $T\left(x_{0}\right)$ is a continuous function of $r$ we conclude that at some intermediate value of $r$ the MFAT attains a minimum value (see Fig. 1). Unfortunately we cannot obtain a close analytical expression for the minimum rate (which, among other variables and parameters, will vary depending on the initial position $x_{0}$ ). This rate is the solution of the transcendental equation, $\partial T / \partial r=0$, which has to be solved numerically in every practical setting. ${ }^{8}$

\footnotetext{
${ }^{7}$ As already mentioned, some results of this work can be obtained by employing different approaches. This is also the case for Eq. (57) which could have been obtained using the first-passage density when $r=0$ (i.e., from the telegraphic process without resettings) following the procedure of Ref. [13].

${ }^{8}$ For diffusion processes and in the special case when $x_{0}=x_{r}$, it has been recently obtained [13] the rate at which $T\left(x_{0}\right)$ is minimum. The procedure involves the numerical solution of a rather simple transcendental equation (see main text below). For telegraphic processes we can do a similar development. However, even in the particular case of $x_{0}=x_{r}$, the transcendental equation to be solved
} 


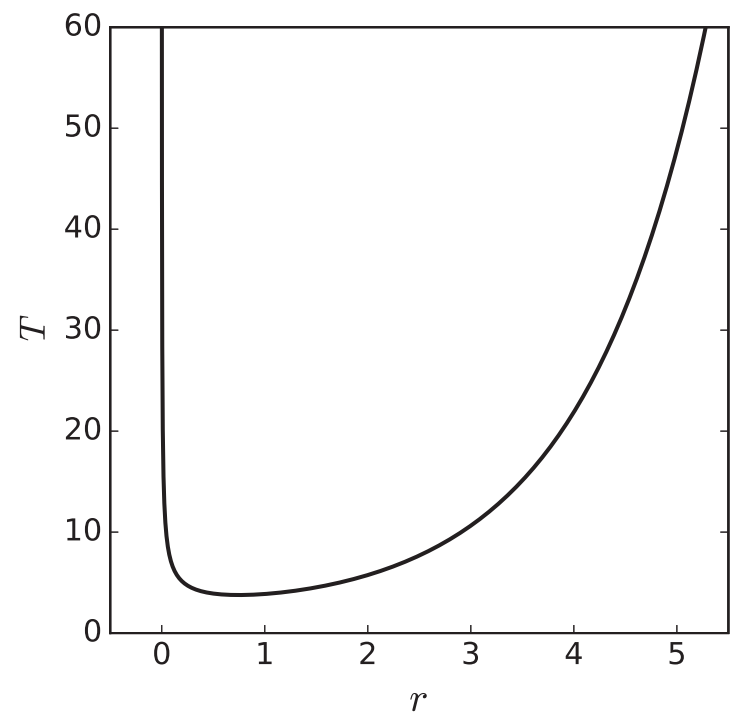

FIG. 1. Representation of $T\left(x_{0}\right)$ in terms of the resetting rate $r$ [cf. Eq. (57)] for parameter values $\lambda=1 / 2,\left|x_{r}-x_{c}\right|=v$, and $\mid x_{0}-$ $x_{c} \mid=2 v$.

\section{Limiting cases}

Let us finish this section by obtaining the expressions for the survival probability and the MFAT in the diffusive and wave limits of the telegraphic process.

\section{Diffusive limit}

The diffusive limit is obtained by setting $\lambda \rightarrow \infty, v \rightarrow$ $\infty$, and $v^{2} /(2 \lambda) \rightarrow D$. In this case $\rho(s)=\sqrt{(r+s) / D}$ and Eqs. (56) and (57) reduce to

$$
\hat{S}\left(s \mid x_{0}\right)=\frac{1-e^{-\left|x_{0}-x_{c}\right| \sqrt{(r+s) / D}}}{s+r e^{-\left|x_{r}-x_{c}\right| \sqrt{(r+s) / D}}}
$$

and

$$
T\left(x_{0}\right)=\frac{1}{r} e^{\left|x_{r}-x_{c}\right| \sqrt{r / D}}\left[1-e^{-\left|x_{0}-x_{c}\right| \sqrt{r / D}}\right] .
$$

Expanding Eq. (62) for small values of $r$ we see that the MFAT diverges like the general telegraphic case [cf. Eq. (59)]

$$
T\left(x_{0}\right) \simeq \frac{1}{\sqrt{r}}\left|x_{0}-x_{c}\right| / \sqrt{D} \quad(r \rightarrow 0) .
$$

For large values of the resetting rate we see from Eq. (62) that

$$
T\left(x_{0}\right) \sim \frac{1}{r} e^{\left|x_{r}-x_{c}\right| / \sqrt{r / D}} \quad(r \rightarrow \infty),
$$

proving that in the diffusion limit $T\left(x_{0}\right)$ grows more slowly than the telegraphic case given by Eq. (60) (see also Fig. 2).

We therefore see that, as in the general case described by Eq. (57), the MFAT is not a monotonous function of $r$ and $T\left(x_{0}\right)$ reaches a minimum value for a particular resetting frequency $r_{\min }$ which has to be obtained numerically for each set of parameters $D, x_{0}, x_{r}$, and $x_{r}$.

is more complicated and depends on the parameters $\lambda, v$ and also on $\left|x_{0}-x_{c}\right|$ with the subsequent lack of appeal and generality. We do not present here such a development.

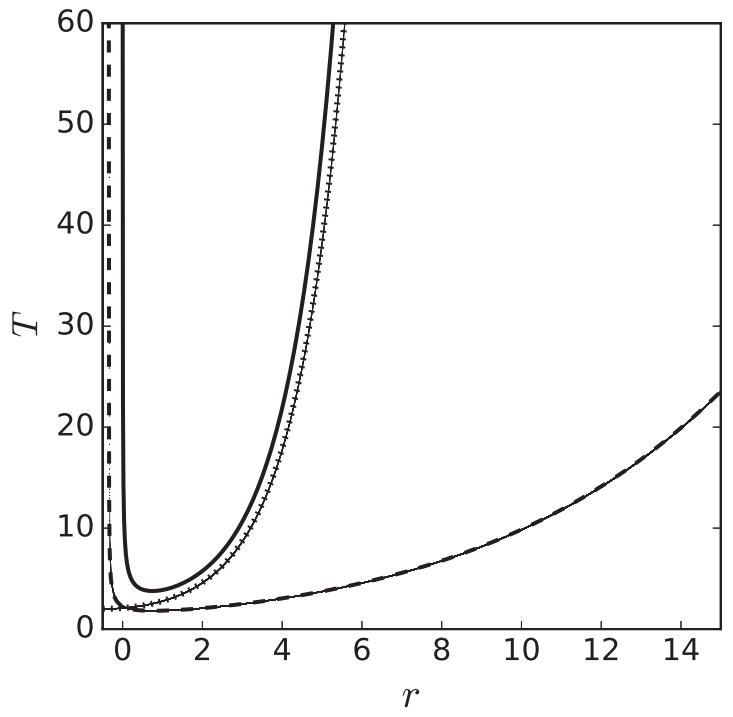

FIG. 2. Representation of $T\left(x_{0}\right)$ in terms of the resetting rate $r$. (i) Solid line: telegraphic process, Eq. (57), parameter values $\lambda=$ $1 / 2,\left|x_{r}-x_{c}\right|=v$, and $\left|x_{0}-x_{c}\right|=2 v$. (ii) Dashed line: diffusion limit, Eq. (62), parameter values $\left|x_{r}-x_{c}\right|=\sqrt{D}$ and $\left|x_{0}-x_{c}\right|=$ $2 \sqrt{D}$. (iii) Dotted line: wave limit, Eq. (66), with $\left|x_{r}-x_{c}\right|=v$ and $\left|x_{0}-x_{c}\right|=2 v$.

In the special case when resetting coincides with the initial position, $x_{r}=x_{0}$, we see from Eq. (62) that MFAT reduces to

$$
T\left(x_{0}\right)=\frac{1}{r}\left[e^{\left|x_{0}-x_{c}\right| \sqrt{r / D}}-1\right],
$$

which agrees with a previous result [3]. The rate at which $T\left(x_{0}\right)$ is minimum is the solution to $\partial T / \partial r=0$. In the particular case given in Eq. (64) one can easily show that the minimum rate is given by $r_{\min }=\xi^{2} D /\left(x_{0}-x_{c}\right)^{2}$, where $\xi=1.594 \ldots$ is the nonzero solution of the transcendental equation $e^{-\xi}=1-\xi / 2[13]$.

\section{Wave limit}

For the wave limit $\lambda \rightarrow 0$ and $\rho(s)=(r+s) / v$. Equations (56) and (57) now reduce to

$$
\hat{S}\left(s \mid x_{0}\right)=\frac{1-e^{-(r+s)\left|x_{0}-x_{c}\right| / v}}{s+r e^{-(r+s)\left|x_{r}-x_{c}\right| / v}}
$$

and

$$
T\left(x_{0}\right)=\frac{1}{r} e^{r\left|x_{r}-x_{c}\right| / v}\left[1-e^{-r\left|x_{0}-x_{c}\right| / v}\right] .
$$

Contrary to the general case given in Eq. (57) we will see that in the wave limit the MFAT is an increasing function of $r$. Let us first observe that in this limiting case $T\left(x_{0}\right)$ does not diverge when $r \rightarrow 0$ but goes to the finite value: ${ }^{9}$

$$
T\left(x_{0}\right) \rightarrow \frac{\left|x_{0}-x_{c}\right|}{v} \quad(r \rightarrow 0),
$$

${ }^{9}$ When $r=0$ Eq. $(65)$ yields $\hat{S}\left(s \mid x_{0}\right)=\left[1-e^{-s\left|x_{0}-x_{c}\right| / v}\right]$ and in real time reads

$$
S\left(t \mid x_{0}\right)=\Theta\left[\frac{\left|x_{0}-x_{c}\right|}{v}-t\right]
$$


which is the ballistic time. When $r \rightarrow \infty$ the MFAT diverges exponentially:

$$
T\left(x_{0}\right) \sim \frac{1}{r} e^{r\left|x_{r}-x_{c}\right| / v} \quad(r \rightarrow \infty),
$$

which is the same growth as that of the general telegraphic case, Eq. (60). Now the monotonous increasing character of $T$ with $r$ can be seen by taking the derivative of Eq. (66) with respect to $r$. This yields

$$
\frac{\partial T}{\partial r} \rightarrow \frac{1}{v^{2}}\left|x_{0}-x_{c}\right|\left|x_{r}-x_{c}\right|>0 \quad(r \rightarrow 0)
$$

and

$$
\frac{\partial T}{\partial r} \sim \frac{1}{r v}\left|x_{r}-x_{c}\right| e^{\left|x_{r}-x_{c}\right| r / v}>0 \quad(r \rightarrow \infty),
$$

thus proving that the MFAT is a monotonous increasing function of $r$ (see Fig. 2). In fact the minimum value of $T\left(x_{0}\right)$ is attained when $r=0$. In other words, the ballistic time is the minimum MFAT.

Let us briefly mention a relatively hidden aspect of the problem: the fact that, as far as the MFAT is concerned, the limits $r \rightarrow 0$ and $\lambda \rightarrow 0$ do not commute. Indeed, (i) let us first note that the limit $r \rightarrow 0$ (for any $\lambda \neq 0$ ) corresponds to a situation where the telegraphic process is undergoing less and less resettings. When we finally set $r=0$, the combined process becomes a homogeneous telegraphic process for which the MFAT is infinite, even for arbitrarily small values of $\lambda$ [see Eq. (59)]. (ii) Secondly, suppose that $\lambda \rightarrow 0$ (for any $r \neq 0$ ) which corresponds to a wave process with the addition of resettings. In the limit $\lambda=0$ and as far as $r \neq 0$ we get an inhomogeneous telegraphic process [cf. Eq. (43)]. In this case, and because of the inhomogeneity (which is due to resettings) the MFAT is not infinite but given by Eq. (66) which approaches the ballistic time, Eq. (67), for arbitrarily small values of $r$ (see also footnote 9). Therefore the limits $r \rightarrow 0$ (reset-free limit) and $\lambda \rightarrow 0$ (wave limit) do not commute. Let us finally note that when $\lambda=0$ and $r=0$ simultaneously, the combined process corresponds to a free wave process. In this case, as can be seen in Eq. (8), the propagator is given by deterministic pulses moving in opposite directions and the time for reaching any location is ballistic.

We finally comment on another aspect of resetting that consists in studying the effect of the addition of a drift on the resetting mechanism. Obviously such an effect will depend on the precise nature of the drift. As far as the effects on the stationary distribution, this has been studied in [27] for constant and linear drifts. In a very recent work [52] it has been shown that the addition of a (constant) drift to a diffusion process with Poissonian resettings alters the MFAT in the sense that, depending on the location of the target and the sign of the drift, the MFAT becomes bigger or smaller than the MFAT when no drift is present. In other words, the addition of a drift may or may not help in the search process. One may expect the same behavior for telegraphic processes, because,

which is the survival probability of a deterministic wave process [compare with Eq. (8)]. The MFAT can now be obtained substituting this expression into Eq. (47) which yields the ballistic time (67). as shown in Sec. II, any telegraphic process has a strong diffusive component. In any case this can be seen quantitatively in the case of a constant drift, $f(x)=\mu$, with $x_{0}=x_{r}=0$. One can easily show in this case that for a positive drift, $\mu>0$, the MFAT is a decreasing function of the drift for a positive target and an increasing one for negative targets. We will try to further develop this issue in a future work. ${ }^{10}$

\section{CONCLUDING REMARKS}

In this paper we have analyzed the problem when telegraphic processes are under a resetting mechanism which, after random intervals of time, brings the process to a fixed position. We have assumed that times at which reset takes place are Poissonian. The combination of random motion with stochastic resetting has many practical applications notably in searching processes such as animal foraging, protein search, and computer-aide web searches, among many others.

We have obtained the modifications to the ordinary telegrapher's equation when the process is under Poissonian resetting events, not only for the forward equation (24),

$$
\frac{\partial^{2} p}{\partial t^{2}}+2(\lambda+r) \frac{\partial p}{\partial t}=v^{2} \frac{\partial^{2} p}{\partial x^{2}}+r(r+2 \lambda)\left[\delta\left(x-x_{r}\right)-p\right]
$$

but for the backward equation (39) as well,

$$
\frac{\partial^{2} p}{\partial t^{2}}+2(\lambda+r) \frac{\partial p}{\partial t}=v^{2} \frac{\partial^{2} p}{\partial x_{0}^{2}}+r \frac{\partial p^{(r)}}{\partial t}+r(r+2 \lambda)\left[p^{(r)}-p\right],
$$

where $p^{(r)}=p\left(x, t \mid x_{r}\right)$ and $x_{r}$ is the resetting position. It is worth noticing the analogies and differences among both equations.

Likewise, for diffusion processes we have proved that stochastic resettings also stabilize telegraphic processes, in the sense that the combined processes are stationary while the reset-free process is not. The stationary distribution is the tent-shape density (27),

$$
p_{\mathrm{st}}(x)=\frac{1}{2 v} \sqrt{r^{2}+2 \lambda r} \exp \left\{-\left|x-x_{r}\right| \sqrt{r^{2}+2 \lambda r} / v\right\},
$$

showing, the otherwise intuitive fact, that resetting mechanisms stabilize telegraphic processes around the average value given by the resetting position $x_{r}$.

The backward equation has allowed us to study the firstarrival problem, one of the cornerstones of resetting. We have thus been able to obtain the exact expression for the mean first-arrival time from initial position $x_{0}$ to some threshold $x_{c}$,

$$
T\left(x_{0}\right)=\frac{1}{r} e^{\rho_{0}\left|x_{r}-x_{c}\right|}\left[1-e^{-\rho_{0}\left|x_{0}-x_{c}\right|}\right],
$$

where $\rho_{0}$ is given in Eq. (58).

As in diffusion processes, the resetting mechanism optimizes the combined process; in the sense that the process becomes stationary and the mean first-arrival time $T\left(x_{0}\right)$ to some fixed critical value is greatly optimized because $T\left(x_{0}\right)$ attains a minimum value for a particular value of the resetting rate. In other words, there exists an optimal resetting rate that

\footnotetext{
${ }^{10}$ All of this can be extended to a linear drift; however, studying the effect of a general nonlinear drift seems to be beyond reach.
} 
may considerably diminish the search time in any searching process.

We have shown that these characteristics, which had been first obtained for diffusion processes, remain valid for telegraphic processes but now depending on two parameters (propagation speed and characteristic time) which modulate the entire response of the system from diffusionlike to wavelike behavior. All of this sustaining the universal character of the resetting mechanism.

\section{ACKNOWLEDGMENTS}

Partial financial support from MINECO under Contract No. FIS2016-78904-C3-2-P and from AGAUR under Contract No. 2017SGR1064 is acknowledged. Fruitful discussions with Josep Llosa and Miquel Montero are warmly acknowledged, as well as to Maria Masoliver for drawing Figs. 1 and 2. I thank an anonymous referee for indicating the works [36] and [37] and also for pointing out that the addition of a drift may or may not help in the search process.

\section{APPENDIX A: SOLUTION TO THE TELEGRAPHER'S EQUATION}

The first step in solving TE (3) with initial conditions (5) consists in taking the joint Fourier-Laplace transform:

$$
\hat{\tilde{p}}_{0}\left(\omega, s \mid x_{0}\right)=\int_{-\infty}^{\infty} e^{i \omega x} d x \int_{0}^{\infty} e^{-s t} p_{0}\left(x, t \mid x_{0}\right) d t
$$

This transformation turns the initial-value problems (3) and (5) into an algebraic equation, whose solution is straightforward and reads [38,39]

$$
\hat{\tilde{p}}_{0}\left(\omega, s \mid x_{0}\right)=\frac{(s+2 \lambda) e^{i \omega x_{0}}}{s^{2}+2 \lambda s+v^{2} \omega^{2}} .
$$

Fourier inverting and taking into account that

$$
\mathcal{F}^{-1}\left\{\frac{a e^{i \omega x_{0}}}{b^{2}+c^{2} \omega^{2}}\right\}=\frac{a}{2|b||c|} e^{-\left|x-x_{0}\right||b| /|c|},
$$

we have

$$
\hat{p}_{0}\left(x, s \mid x_{0}\right)=\frac{s+2 \lambda}{2 v \sqrt{s^{2}+2 \lambda s}} \exp \left\{-\left|x-x_{0}\right| \sqrt{s^{2}+2 \lambda s} / v\right\} .
$$

We rewrite this equation as

$$
\begin{aligned}
\hat{p}_{0}\left(x, s \mid x_{0}\right)= & \frac{(s+\lambda)+\lambda}{2 v \sqrt{(s+\lambda)^{2}-\lambda^{2}}} \\
& \times \exp \left\{-\left|x-x_{0}\right| \sqrt{(s+\lambda)^{2}-\lambda^{2}} / v\right\}
\end{aligned}
$$

and take into account the well-known property of the Laplace transform,

$$
\mathcal{L}^{-1}\{\hat{f}(s+\lambda)\}=e^{-\lambda t} \mathcal{L}^{-1}\{\hat{f}(s)\},
$$

where $\mathcal{L}^{-1}\{\cdot\}$ stands for the inverse Laplace transform. We thus have

$$
p_{0}\left(x, t \mid x_{0}\right)=\frac{1}{2 v} e^{-\lambda t} \mathcal{L}^{-1}\left\{\frac{s+\lambda}{\sqrt{s^{2}-\lambda^{2}}}\right.
$$

$$
\left.\times \exp \left[-\left|x-x_{0}\right| \sqrt{s^{2}-\lambda^{2}} / v\right]\right\} .
$$

We next use the following inversion formulas [48]:

$$
\mathcal{L}^{-1}\left\{\frac{e^{-b\left(s^{2}-a^{2}\right)^{1 / 2}}}{\left(s^{2}-a^{2}\right)^{1 / 2}}\right\}=\Theta(t-b) I_{0}\left[a\left(t^{2}-b^{2}\right)^{1 / 2}\right]
$$

and

$$
\begin{aligned}
\mathcal{L}^{-1}\left\{\frac{s e^{-b\left(s^{2}-a^{2}\right)^{1 / 2}}}{\left(s^{2}-a^{2}\right)^{1 / 2}}\right\}= & \delta(t-b)+\Theta(t-b) \frac{a t}{\left(t^{2}-b^{2}\right)^{1 / 2}} \\
& \times I_{1}\left[a\left(t^{2}-b^{2}\right)^{1 / 2}\right],
\end{aligned}
$$

where $I_{0}(\cdot)$ and $I_{1}(\cdot)$ are modified Bessel functions.

Substituting these inverse transforms into the right-hand side of Eq. (A3) with the identifications $a=\lambda$ and $b=\mid x-$ $x_{0} \mid / v$ we readily get the solution given in Eq. (6).

\section{APPENDIX B: THE INHOMOGENEOUS TELEGRAPHER'S EQUATION}

Let us prove that the propagator $p\left(x, t \mid x_{0}\right)$ of the combined process obeys the inhomogeneous TE (24). Multiplying Eq. (21) by $2 \lambda$, adding Eq. (22), subtracting Eq. (23) multiplied by $v^{2}$ and reorganizing terms, we get

$$
\begin{aligned}
\frac{\partial^{2} p}{\partial t^{2}} & +2(\lambda+r) \frac{\partial p}{\partial t}-v^{2} \frac{\partial^{2} p}{\partial x^{2}} \\
= & e^{-r t}\left[\frac{\partial^{2} p_{0}}{\partial t^{2}}+2 \lambda \frac{\partial p_{0}}{\partial t}-v^{2} \frac{\partial^{2} p_{0}}{\partial x^{2}}\right]+r \frac{\partial p^{(r)}}{\partial t} \\
& +r(r+2 \lambda)\left[p^{(r)}-p\right]-v^{2} r \int_{0}^{t} e^{-r t^{\prime}} \frac{\partial^{2} p_{0}^{(r)}}{\partial x^{2}} d t^{\prime},
\end{aligned}
$$

but [see Eq. (15)]

$$
\frac{\partial^{2} p_{0}}{\partial t^{2}}+2 \lambda \frac{\partial p_{0}}{\partial t}-v^{2} \frac{\partial^{2} p_{0}}{\partial x^{2}}=0
$$

hence

$$
\begin{aligned}
& \frac{\partial^{2} p}{\partial t^{2}}+2(\lambda+r) \frac{\partial p}{\partial t}-v^{2} \frac{\partial^{2} p}{\partial x^{2}} \\
& \quad=r \frac{\partial p^{(r)}}{\partial t}+r(r+2 \lambda)\left[p^{(r)}-p\right]-v^{2} r \int_{0}^{t} e^{-r t^{\prime}} \frac{\partial^{2} p_{0}^{(r)}}{\partial x^{2}} d t^{\prime}
\end{aligned}
$$

On the other hand, setting $x_{0}=x_{r}$ in the TE (B1) for a reset-free propagator we have

$$
\frac{\partial^{2} p_{0}^{(r)}}{\partial x^{2}}=\frac{1}{v^{2}} \frac{\partial^{2} p_{0}^{(r)}}{\partial t^{2}}+\frac{2 \lambda}{v^{2}} \frac{\partial p_{0}^{(r)}}{\partial t}
$$

and

$$
\begin{aligned}
v^{2} \int_{0}^{t} e^{-r t^{\prime}} \frac{\partial^{2} p_{0}^{(r)}}{\partial x^{2}} d t^{\prime}= & \int_{0}^{t} e^{-r t^{\prime}} \frac{\partial^{2} p_{0}^{(r)}}{\partial t^{\prime 2}} d t^{\prime} \\
& +2 \lambda \int_{0}^{t} e^{-r t^{\prime}} \frac{\partial p_{0}^{(r)}}{\partial t^{\prime}} d t^{\prime}
\end{aligned}
$$


Integrating by parts

$$
\int_{0}^{t} e^{-r t^{\prime}} \frac{\partial p_{0}^{(r)}}{\partial t^{\prime}} d t^{\prime}=e^{-r t} p_{0}^{(r)}-p\left(x, 0 \mid x_{r}\right)+r \int_{0}^{t} e^{-r t^{\prime}} p_{0}^{(r)} d t^{\prime},
$$

but $p\left(x, 0 \mid x_{r}\right)=\delta\left(x-x_{r}\right)$ and [cf. Eq. (17)]

$$
p^{(r)}=e^{-r t} p_{0}^{(r)}+\int_{0}^{t} e^{-r t^{\prime}} p_{0}^{(r)} d t^{\prime}
$$

Hence

$$
\int_{0}^{t} e^{-r t^{\prime}} \frac{\partial p_{0}^{(r)}}{\partial t^{\prime}} d t^{\prime}=p^{(r)}-\delta\left(x-x_{r}\right)
$$

As to the second derivative term with respect to time in Eq. (B3), integrating also by parts we have

$$
\begin{aligned}
\int_{0}^{t} e^{-r t^{\prime}} \frac{\partial^{2} p_{0}^{(r)}}{\partial t^{\prime 2}} d t^{\prime}= & e^{-r t} \frac{\partial p_{0}^{(r)}}{\partial t}-\left.\frac{\partial p_{0}^{(r)}}{\partial t}\right|_{t=0} \\
& +r \int_{0}^{\infty} e^{-r t^{\prime}} \frac{\partial p_{0}^{(r)}}{\partial t^{\prime}} d t^{\prime}
\end{aligned}
$$

but [cf. Eq. (16)] $\partial p_{0}^{(r)} /\left.\partial t\right|_{t=0}=0$ and using Eq. (B4) we get

$$
\int_{0}^{t} e^{-r t^{\prime}} \frac{\partial^{2} p_{0}^{(r)}}{\partial t^{\prime 2}} d t^{\prime}=e^{-r t} \frac{\partial p_{0}^{(r)}}{\partial t}+r\left[p^{(r)}-\delta\left(x-x_{r}\right)\right] .
$$

On the other hand, setting $x_{0}=x_{r}$ in Eq. (21) we see that

$$
\frac{\partial p^{(r)}}{\partial t}=e^{-r t} \frac{\partial p_{0}^{(r)}}{\partial t} \text {. }
$$

Hence

$$
\int_{0}^{t} e^{-r t^{\prime}} \frac{\partial^{2} p_{0}^{(r)}}{\partial t^{\prime 2}} d t^{\prime}=\frac{\partial p^{(r)}}{\partial t}+r\left[p^{(r)}-\delta\left(x-x_{r}\right)\right] .
$$

Plugging (B4) and (B5) into (B3) yields

$v^{2} \int_{0}^{t} e^{-r t^{\prime}} \frac{\partial^{2} p_{0}^{(r)}}{\partial x^{2}} d t^{\prime}=\frac{\partial p^{(r)}}{\partial t}+(r+2 \lambda)\left[p^{(r)}-\delta\left(x-x_{r}\right)\right]$, which substituting into Eq. (B2) results, after some cancellations, in TE (24):

$$
\frac{\partial^{2} p}{\partial t^{2}}+2(\lambda+r) \frac{\partial p}{\partial t}=v^{2} \frac{\partial^{2} p}{\partial x^{2}}+r(r+2 \lambda)\left[\delta\left(x-x_{r}\right)-p\right] .
$$

[1] S. C. Manrubia and D. H. Zanette, Phys. Rev. E 59, 4945 (1999).

[2] M. Montero, A. Masó-Puigdellosas, and J. Villarroel, Eur. Phys. J. B 90, 176 (2017).

[3] M. R. Evans and S. N. Majumdar, Phys. Rev. Lett. 106, 160601 (2011).

[4] M. R. Evans and S. N. Majumdar, J. Phys. A 44, 435001 (2011).

[5] M. R. Evans, S. N. Majumdar, and K. Maelick, J. Phys. A 46, 185001 (2013).

[6] J. Whitehouse, M. R. Evans, and S. N. Majumdar, Phys. Rev. E 87, 022118 (2013).

[7] M. R. Evans and S. N. Majumdar, J. Phys. A 49, 225001 (2014).

[8] S. Gupta, S. N. Majumdar, and G. Schehr, Phys. Rev. Lett. 112, 220601 (2014).

[9] A. Pal, A. Kundu, and M. R. Evans, J. Phys. A 49, 225001 (2016).

[10] M. Montero and J. Villarroel, Phys. Rev. E 87, 012116 (2013).

[11] M. Montero and J. Villarroel, Phys. Rev. E 94, 032132 (2016).

[12] L. Kuśmierz and E. Gudowska-Nowak, Phys. Rev. E 92, 052127 (2015).

[13] S. Reuveni, Phys. Rev. Lett. 116, 170601 (2016).

[14] S. Belan, Phys. Rev. Lett. 120, 080601 (2018).

[15] T. S. Biró and J. Néda, Physica A (Amsterdam) 499, 335 (2018).

[16] T. Rotbart, S. Reuveni, and M. Urbakh, Phys. Rev. E 92, 060101 (2015).

[17] A. Pal and S. Reuveni, Phys. Rev. Lett. 118, 030603 (2017).

[18] A. Chechkin and I. M. Sokolov, Phys. Rev. Lett. 121, 050601 (2018).

[19] A. Badrisarayanan, T. B. Le, and M. T. Laub, J. Cell Biol. 210, 385 (2015).

[20] S. Reuveni, M. Urbalch, and J. Klafter, Proc. Natl. Acad. Sci. USA 111, 4391 (2014).

[21] E. Roldán, A. Lisica, D. Sánchez-Taltavull, and S. W. Grill, Phys. Rev. E 93, 062411 (2016).
[22] V. Méndez, D. Campos, and F. Bartumeus, Stochastic Foundations in Movement Ecology: Anomalous Diffusion, Front Propagation and Random Searches (Springer-Verlag, Berlin, 2013).

[23] A. Reynolds, Phys. Life Rev. 14, 59 (2015).

[24] M. Luby, A. Sinclair, and D. Zuckerman, Inf. Proc. Lett. 47, 173 (1993).

[25] A. Montanari and R. Zecchina, Phys. Rev. Lett. 88, 178701 (2002).

[26] M. Tong, C. Faloutsos, and J.-Y. Pau, in Proceedings of the Sixth International Conference on Data Mining (IEEE Computer Society, Hong Kong, 2006).

[27] A. Pal, Phys. Rev. E 91, 012113 (2015).

[28] M. Shlesinger, J. Klafter, and G. Zumofen, Fractals 03, 491 (1995).

[29] J. Masoliver and G. H. Weiss, Eur. J. Phys. 17, 190 (1996).

[30] D. J. Durian and J. Rudnick, J. Opt. Soc. Am. A 14, 235 (1997).

[31] G. H. Weiss, Physica A (Amsterdam) 311, 381 (2002).

[32] J. B. Keller, Proc. Natl. Acad. Sci. USA 101, 1120 (2004).

[33] D. D. Joseph and L. Preziosi, Rev. Mod. Phys. 61, 41 (1989); 62, 375 (1990).

[34] D. Jou, J. Casas-Vázquez, and G. Lebon, Extended Irreversible Thermodynamics, 4th ed. (Springer, Berlin, 2010).

[35] V. Méndez, D. Campos, and W. Horsthemke, Phys. Rev. E 90, 042114 (2014).

[36] J. Fuchs, S. Gold, and U. Seifert, Eur. Phys. Lett. 113, 60009 (2016).

[37] A. Pal and S. Rahav, Phys. Rev. E 96, 062135 (2017).

[38] J. Masoliver, K. Lindenberg, and G. H. Weiss, Physica A (Amsterdam) 157, 891 (1989).

[39] J. Masoliver and K. Lindenberg, Eur. Phys. J. B 90, 107 (2017). 
[40] J. Masoliver, Phys. Rev. E 96, 022101 (2017).

[41] P. C. De Jagher, Physica A (Amsterdam) 101, 629 (1980).

[42] G. Zumofen and J. Klafter, Phys. Rev. E 47, 851 (1993).

[43] R. Metzler and A. Compte, Physica A (Amsterdam) 268, 454 (1998).

[44] M. A. Olivares-Robles and L. S. Garcia-Colin, Phys. Rev. E 50, 2451 (1994).

[45] L. S. Garcia-Colin and M. A. Olivares-Robles, Physica A (Amsterdam) 220, 165 (1995).

[46] M. A. Olivares-Robles and L. S. Garcia-Colin, J. Non-Equilib. Thermodyn. 21, 361 (1996).
[47] J. Masoliver, Random Processes, First-Passage and Escape (World Scientific, Singapore, 2018).

[48] G. E. Roberts and H. Kaufman, Table of Laplace Transforms (W. B. Saunders, Philadelphia, 1966).

[49] J. Masoliver and G. H. Weiss, Physica A (Amsterdam) 183, 537 (1992).

[50] S. Redner, A Guide to First-Passage Processes (Cambridge University Press, Cambridge, England, 2001).

[51] G. H. Weiss, J. Stat. Phys. 37, 325 (1984).

[52] A. Pal, I. Eliazar, and S. Reuveni, arXiv:1807.09363v3 [condmat.stat-mech]. 